\title{
MRS Workshop Explores the Wonderful World of MEMS Materials
}

The Materials Research Society (MRS) MEMS Materials Issues workshop was held in San Francisco on April 5-6, 2002, immediately after the 2002 MRS Spring Meeting. Organized by Ainissa G. Ramirez of Agere Systems/Bell Labs-Lucent Technologies and Richard P. Vinci of Lehigh University, it presented work from an international group of 12 scientists from industry and academia who are key players in the field of microelectromechanical systems (MEMS) materials. Among the 80 workshop attendees were academics, researchers, and engineers.

Stuart Brown of Exponent set the tone for the event by noting, in his overview, that there are plenty of interesting problems in MEMS. He said that this is good news for those in academia and bad news for those in industry. He remarked that the effects of scale in MEMS give rise to many unknowns in the behavior of crack growth, creep, contamination effects, and wear. These are topics that researchers are exploring, but much still needs to be done. Roya Maboudian of the University of California-Berkeley has undertaken a surface chemistry approach on the issue of release stiction, that is, the adhesion of freestanding structures pulled down by capillary forces in the release step. She demonstrated that the impact of stiction can be diminished with self-assembled monolayer-coated surfaces, which generate greater contact angles with liquids than $\mathrm{SiO}_{2} / \mathrm{Si}$ interfaces.

The crux of the workshop was to profile the materials of MEMS. Speakers demonstrated that MEMS has a greater materials palette than the usual suspects of microelectronics. These new materials include diamond-like carbon, TiNi shape-memory alloys, magnetic alloys, piezoelectric materials, and biomaterials, with each having a unique set of challenges and opportunities. New materials have also been engineered for MEMS, such as MultiPoly ${ }^{\mathrm{TM}}$, presented by Arthur Heuer of Case Western Reserve University (see figure 1). This material takes advantage of tailorability of residual stresses of polysilicon from compressive to tensile stress by modifying the substrate temperature during deposition from $615^{\circ} \mathrm{C}$ to $570^{\circ} \mathrm{C}$, respectively. Using laminates of compressive and tensile layers, long aspect-ratio structures can be generated. This work tackles the issues of stress gradients common in these structures and underscored the quintessential need for a materials understanding in MEMS in order to create novel and reliable structures.

Wolé Soboyejo of Princeton University said that common microelectronic mate-
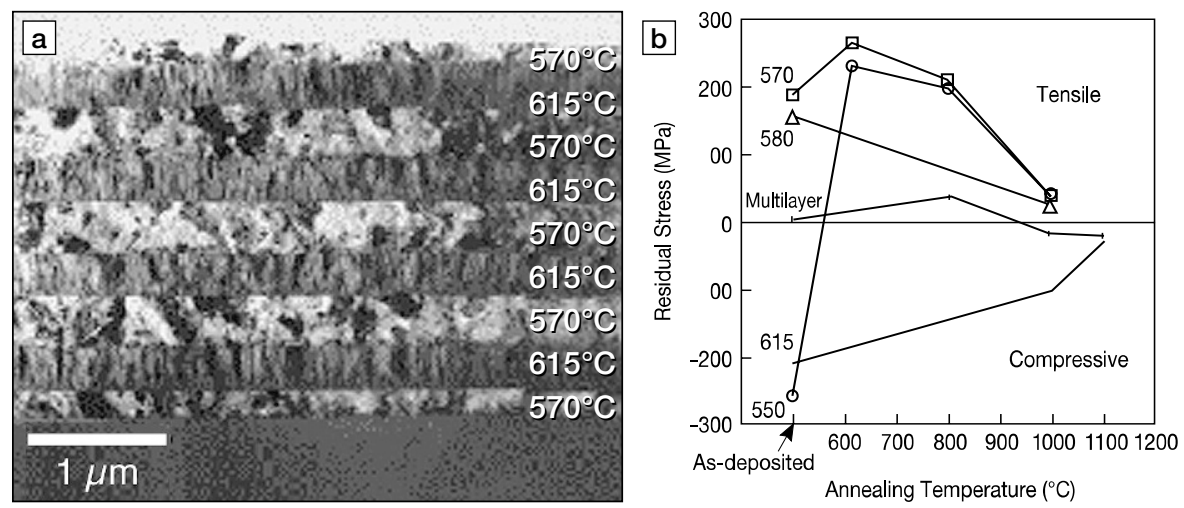

Figure 1. (a) A multilayer laminate of tensile $\left(570^{\circ} \mathrm{C}\right)$ and compressive $\left(615^{\circ} \mathrm{C}\right)$ polysilicon films enables long microelectromechanical systems (MEMS) structures. (b) The observation of the tailorability of film stress underscores the impact of materials understanding in MEMS. Reproduced with permission from J. Yang, H. Kahn, A.-Q. He, S.M. Phillips, and A.H. Heuer, J. Microelectromech. Syst. 9 (2000) p. 485. rials are not biocompatible; for example, nickel and silicon are toxic in the body and will be rejected, which can lead to swelling. Only a few materials are compatible with the body, such as titanium, zirconium, and carbon, but their integration requires further understanding of their morphology and their interplay with cells. Soboyejo also noted that reliability is tantamount in bio-MEMS. For example, if a heart beats at 72 beats per minute, then that converts to 1 billion cycles in 30 years. A MEMS device must far surpass that life cycle to have any utility in the body.

Hoo-Jeong Lee of Agere Systems illuminated that although there are many unknowns in MEMS, our knowledge of bulk materials does not fully forsake us. He paralleled his observations of mechanical testing of aluminum freestanding films to that of the physics of sheet metals. Lee

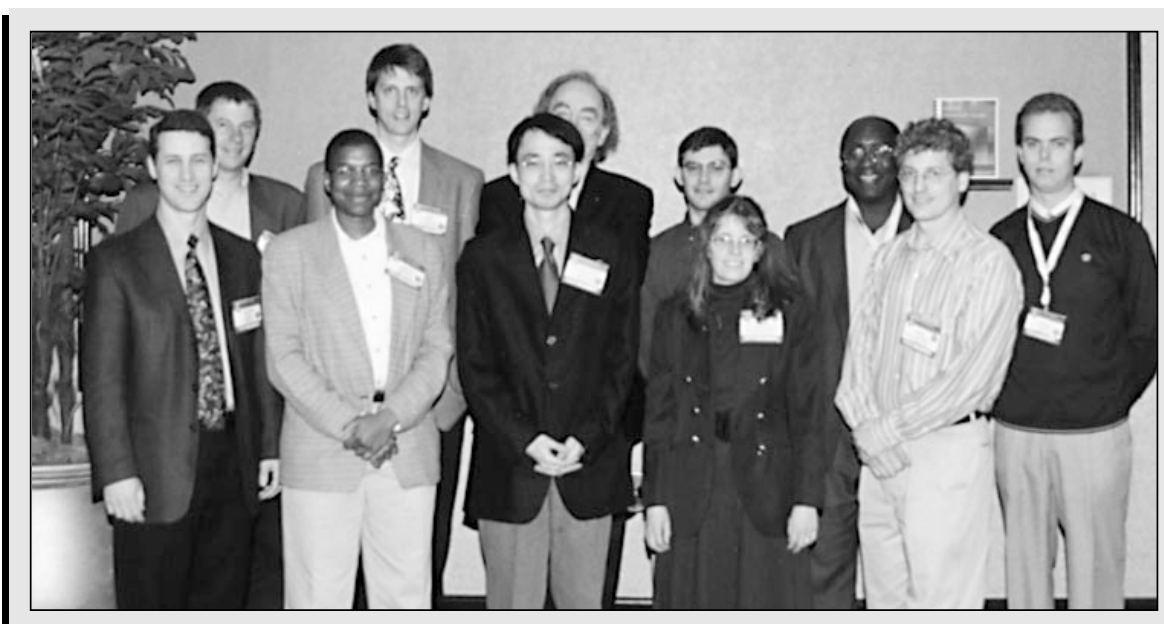

Organizers of the MRS Workshop on MEMS Materials Issues and speakers. First row (left to right): Organizer Richard P. Vinci (Lehigh University) and Organizer Ainissa G. Ramirez (Agere Systems/Bell Labs-Lucent), Hoo-Jeong Lee (Agere Systems), Susan Trolier-McKinstry (The Pennsylvania State University), Thomas Friedmann (Sandia National Laboratories); second row (left to right): Oliver Kraft (Max Planck Institute), Jack Judy (University of CaliforniaLos Angeles), Arthur Heuer (Case Western Reserve University), Herb Shea (Bell Labs/Lucent Technologies), Wolé Soboyejo (Princeton University), and David LaVan (Massachusetts Institute of Technology). Not shown: Roya Maboudian (University of California-Berkeley) and Stuart Brown (Exponent). 


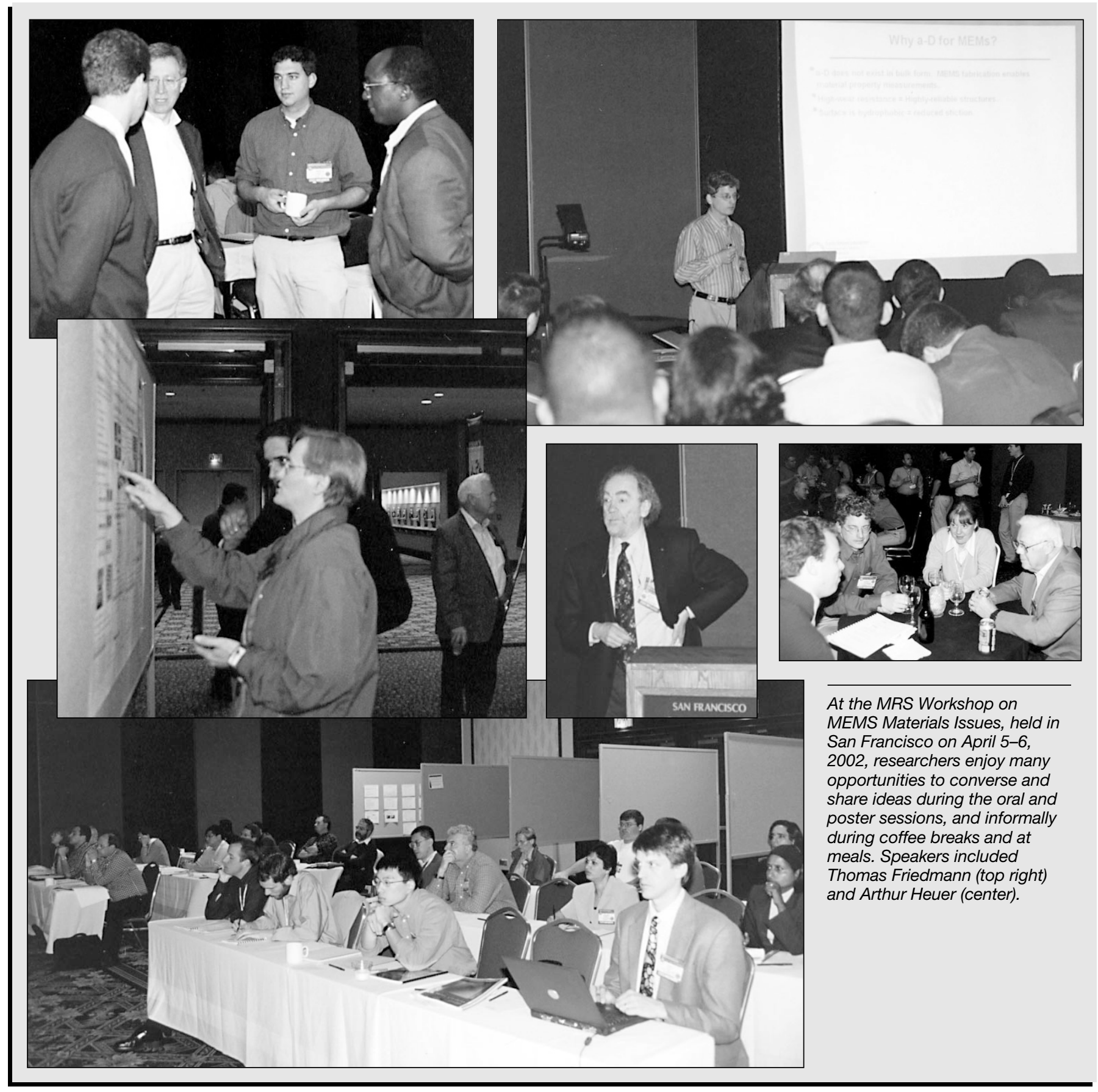

found that the localized deformation extended across the aluminum beam in a manner similar to a diffuse neck in sheet metals. These observations may provide insight into the behavior of the small scale.

With a panel discussion and a poster session of 12 presentations, the two-day workshop enabled an active exchange of ideas. The panel discussed a host of topics that included MEMS standardization and MEMS work in Europe. The topics of the posters included work in the use of polycrystalline silicon germanium in MEMS, nanodiamond, bulge testing of aluminum alloys, and the fabrication of MEMS structures at low temperature on alternate substrates. S.K. Koh and coworkers of the Korea Institute of Science and Technology presented particularly intriguing work of surface modification using ion beams. With this technique, the scientists were able to generate a loudspeaker made from a sheet of plastic. Scientists huddled around this prototype as music propelled from the flimsy and flexible source that could be rolled away for easy storage.

Richard Feynman had predicted the possibilities of MEMS, nanotechnology, and devices on the small scale by commenting that there was plenty of room at the bottom. It seems that for the workshop attendees, that was not such a bad place to be.

AINISSA G. RAMIREZ Chair \& Co-organizer 